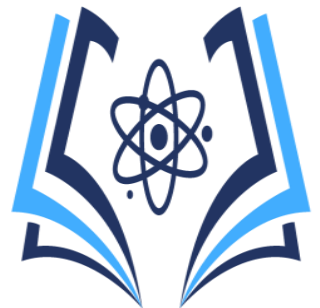

\title{
COVID-19 Ética e Investigación
}

COVID-19 Ethics and Research

\section{COVID-19}

${ }^{1}$ Sidar Edgardo Solórzano Solórzano. Mg, sidar.solorzano@unesum.edu.ec

${ }^{2}$ Mercedes del Rosario Acuña Acebo. Mg, mercedes.acuna@unesum.edu.ec

${ }^{3}$ Doris Susana Delgado Bernal, doris.delgado@unesum.edu.ec

${ }^{4}$ Delia Georgina Bravo Bonoso. Mg, delia.bravo@unesum.edu.ec

Universidad Estatal del Sur de Manabí1,2,3,4

Contacto: sidar.solorzano@unesum.edu.ec

Recibido: 23-08-2021

\section{Resumen}

La crisis sanitaria motivada por la COVID-19 hace necesaria la puesta en marcha, con celeridad, de investigaciones encaminadas a generar evidencias científicas que incidan en el control de sus devastadores efectos. Desde la aparición del primer caso contagiado por el SARS-Cov-2, la pandemia ha experimentado un ascenso en el número de casos casi incontrolable. La producción científica juega un papel clave en la lucha por este necesario control y erradicación. El número de artículos que se produce diariamente desde los inicios sobrepasan los miles a nivel internacional y las editoriales y bases de datos han puesto su mayor esfuerzo para que estas contribuciones tengan la debida prioridad y visibilidad. Este estudio tiene como objetivo caracterizar la producción científica publicadas en revistas indexadas y no indexadas de orden regional e internacional donde se evidencie la gestión ética y calidad investigativa. Materiales y métodos, es un estudio es de tipo observacional, descriptivo, bibliométrico, en artículos sobre COVID-19en base a su ética e investigación, publicados en el periodo enero-2020 a mayo de 2021. Se obtuvo un resultado significativo, el $100 \%$ de los artículos presentó autoría múltiple. Se reportaron 99 autores, de ellos, el $85,85 \%$ de procedencia Internacional; y $14,15 \%$ autorías nacionales. Conclusiones: existe una baja producción científica sobre el tema abarcado a nivel Nacional.

Palabras clave: ser humano, déficit de humanización, empatía e integralidad.

\begin{abstract}
Abstrac
The health crisis caused by COVID-19 makes it necessary to quickly launch research aimed at generating scientific evidence that affects the control of its devastating effects. Since the appearance of the first case infected by SARS-Cov-2, the pandemic has experienced an almost uncontrollable rise in the number of cases. Scientific production plays a key
\end{abstract}


Periodo. Julio - Diciembre 2021

Vol. 5, Nro. 2, Publicado: 2021-12-31

role in the fight for this necessary control and eradication. The number of articles produced daily since the beginning exceeds the thousands internationally and publishers and databases have put their best effort so that these contributions have the due priority and visibility. This study aims to characterize the scientific production published in regional and international indexed and non-indexed journals where ethical management and research quality is evidenced. Materials and methods, it is an observational, descriptive, bibliometric study, in articles about COVID-19 based on its ethics and research, published in the period January-2020 to May 2021. A significant result was obtained, $100 \%$ of the articles presented multiple authorship. 99 authors were reported, of them, $85.85 \%$ of international origin; and $14.15 \%$ national authorship. Conclusions: there is a low scientific production on the subject covered at the National level.

Keywords: human being, humanization deficit, empathy and integrality

\section{Introducción}

Las enfermedades siempre amenazaron a la humanidad. Durante siglos, los seres humanos intentaron comprender sus causas, su proceso de transmisión y prevención con el objetivo de establecer tratamientos y, principalmente, curarlas, consolidando el proceso empírico actual. Hoy se reconoce que la investigación debe seguir el método científico (construir hipótesis, diseñar experimentos, analizar datos y comunicar resultados), además de adaptarse a los principios éticos y bioéticos, para que sus resultados sean validados. Sin embargo, incluso después de siglos de descubrimientos, la naturaleza sorprende constantemente con nuevos virus letales zoonóticos.
La pandemia de COVID-19 nos enfrenta a la urgencia moral de llevar a cabo investigaciones rigurosas tan pronto como sea posible para producir evidencia sobre la seguridad y eficacia de las intervenciones para tratar o prevenir la COVID-19 (1). Sin embargo, al igual que en emergencias anteriores, caracterizadas por una alta mortalidad y la ausencia de tratamientos seguros y eficaces, las intervenciones que no han sido probadas previamente para COVID-19 se están proporcionando fuera de protocolos de investigación con seres humanos. En circunstancias ordinarias, las intervenciones se prueban primero en investigaciones que tienen como objetivo principal producir conocimiento.

La pandemia por COVID-19 ha constituido, a nivel mundial, un desafío para los sistemas de salud debido a que deben enfrentar a un agente desconocido que presenta una alta tasa de transmisión y un comportamiento clínico desconcertante, lo que dificulta la elaboración de políticas sanitarias que permitan un acceso equitativo a la atención médica y una asignación racional de los recursos disponibles. Ya se ha demostrado que muchos dilemas éticos surgen en el manejo de estos pacientes: desde la decisión de a quién se debe tomar pruebas de descarte, la aplicación de tratamientos experimentales o la elección de qué paciente puede acceder a tratamiento especializado (aplicación de ventilación mecánica o internamiento en una unidad de cuidados intensivos)

En el escenario desafiante que se impuso se necesitaron medidas excepcionales para satisfacer la demanda de la población y de la comunidad científica, tanto en el proceso de investigación ética y de integridad como en la publicación de los resultados de la investigación clínica. La naturaleza excepcional del momento hizo que los investigadores 
Periodo. Julio - Diciembre 2021

Vol. 5, Nro. 2, Publicado: 2021-12-31

hicieran grandes esfuerzos para encontrar soluciones terapéuticas y farmacológicas que detengan el virus. la toma de decisiones de emergencia en la búsqueda de tratamientos y soluciones crea un ambiente de incertidumbre y ansiedad que extiende el concepto de vulnerabilidad a todos los pacientes.

Por otra parte, la gran cantidad de investigación clínica y los procesos que apuntan a una aprobación más eficiente y una difusión más amplia y rápida de los resultados generan dudas con relación a los derechos básicos de las personas. En el ámbito legal, varias situaciones llevan a la reflexión sobre los propósitos de la Ley, en particular su función de garantizar la protección de los vulnerables, en resistencia a las presiones políticas, de mercado y las actitudes individualistas

La Organización de la Naciones Unidas para la Educación, Ciencia y la Cultura UNESCO asesora sobre los aspectos éticos relativos a las ciencias de la vida y la salud humana en un sentido integral y asegurar la defensa de la integridad, la dignidad y los derechos humanos de los habitantes de la región, expresa su preocupación ante la realización de investigaciones biomédicas en relación con la pandemia de enfermedad infecciosa por coronavirus Covid-19 (Red de America Latinas Y el Caribe de Comites Nacionales de Bioetica, 2020)

En este contexto de incertidumbre, y con el fin de tomar las mejores decisiones en salud pública, se vuelve un imperativo ético la generación de conocimientos. En el marco de una pandemia, la investigación debe superar diversos retos como el tiempo necesario para la generación de evidencias, el financiamiento para investigaciones clínicas que busquen alternativas terapéuticas, la disponibilidad del personal sanitario -quienes también se encuentran en un dilema al decidir si participar o no de un estudio- $\mathrm{y}$, finalmente, el cumplimiento de las consideraciones éticas en este especial entorno, buscando el balance entre la necesidad de información y el respeto a los derechos de los pacientes afectados y sus familias

La investigación clínica se organiza actualmente en torno a un proceso científico (idealización, ejecución y difusión de resultados) que se basa en la valoración de la persona humana y la protección de los derechos fundamentales. En este sentido, este tipo de investigación se estructuró de manera a salvaguardar, a través de regulaciones e instituciones, el respeto a estrictos estándares éticos, tanto nacionales como internacionales, para garantizar que las personas participantes no se expongan a riesgos, además de garantizar que los datos generados por la investigación sean válidos y precisos. Para comprender este proceso de investigación ética e integridad científica, primero es necesario definir el concepto de investigación clínica y caracterizar sus fases, así como las normas y agentes involucrados (Dadalto, Medeiros Royo, \& Silva Costa, 2020)

La situación actual exige prontitud en la generación de conocimiento científico en torno al COVID-19 que facilite la pronta identificación del comportamiento de la enfermedad, así como el reconocimiento de tratamientos efectivos para dotar al personal sanitario de las herramientas que le permitan paliar los efectos de la pandemia en la población. Sin embargo, aún con el panorama expuesto y con la necesidad imperante de respuestas efectivas y prontas, no se debe perder de vista las consideraciones éticas que validan y dan sustento a la investigación biomédica.

Por otro lado, las investigaciones observacionales también son necesarias para ilustrar a la comunidad científica sobre aspectos relacionados a la 
Periodo. Julio - Diciembre 2021

Vol. 5, Nro. 2, Publicado: 2021-12-31

propagación de la enfermedad, sus manifestaciones clínicas y aspectos epidemiológicos que ayuden a la elaboración de políticas sanitarias eficaces en la población. En estas circunstancias, cobran gran importancia las investigaciones operativas que usan la información proveniente de bases de datos nacionales, usualmente obtenidas al momento de realizar pruebas diagnósticas, las que pueden ser solicitadas en el marco de leyes de transparencia. Aquí es donde se tiene que buscar un contexto en el que la generación de conocimientos no vaya por encima de la confidencialidad de los datos personales de los pacientes.

Si bien es evidente la necesidad de generación de conocimiento, resulta indispensable que el desarrollo de esta se realice en un marco de reconocimiento por los derechos humanos y los pactos y acuerdos internacionales que velan por el resguardo de la protección de los sujetos de investigación. Los comités de ética en investigación tienen probados y estandarizados sus procesos de evaluación; sin embargo, la necesidad mundial de respuesta a este problema de dimensiones globales es primordial; por ello, es justo priorizar la revisión de los proyectos encaminados a la generación de conocimiento de la enfermedad, fármacos efectivos para su tratamiento y formas de erradicación, como vacunas.

La priorización de valoración no debe, en ninguna circunstancia, disminuir el rigor científico y ético con el que se efectúa, ya que este rigor asegura su calidad y aplicabilidad. De ahí que la respuesta a la pregunta planteada de manera previa es: más que acelerar, se requiere priorizar y resolver de manera expedita los procesos de revisión ética. Hasta ahora son diversos los cuestionamientos en torno a los alcances y limitaciones de los consentimientos informados, tanto en la aplicación de la medicina ante un escenario de emergencia como en su uso en los proyectos de investigación

Pautas éticas internacionales para la investigación relacionada con la salud con seres humanos" afirma que "es necesario obtener el consentimiento informado en todas las investigaciones que se lleven a cabo durante las emergencias que involucren participantes humanos o sus muestras o datos identificables. Las pautas estipulan algunas circunstancias en las cuales el comité de revisión ética puede decidir dar una dispensa al requisito de obtener consentimiento informado: a) si no es factible obtenerlo y los estudios b) tienen un importante valor social y c) sólo suponen riesgos mínimos para los participantes", (Consejo de Organizaciones Internacionales de las Ciencias Medicas, 2016) por lo cual resulta indispensable la obtención del consentimiento informado.

El consentimiento informado es un proceso comunicativo en donde se establecen, de común acuerdo, las aplicaciones y los límites de uso de la información que el sujeto de investigación está aportando; por su parte, los consentimientos informados amplios son acuerdos abiertos en los que se permite el uso de datos y muestras biológicas en proyectos de investigación que aún no están diseñados, en pocas palabras es la firma de un acuerdo en blanco; sin embargo, esta información podrá aportar información para la prevención de eventos de estas características.

Esta crisis sanitaria nos ha dado la oportunidad de aprender y reconsiderar varias situaciones, la primera es que cada miembro de la sociedad tiene un papel y un tipo de responsabilidad, y es menester de todos reconocerlo y actuar en pro de ello, como la aportación de información para que la comunidad científica pueda generar su conocimiento; la segunda 
es que las autoridades tienen un papel fundamental pero que sin el apoyo de la sociedad resulta muy complejo llegar a un buen resultado; y la tercera es que las crisis no se pueden superar si no hay de por medio el establecimiento de principios y valores éticos en todos los elementos que contempla la sociedad; valores como la transparencia, la equidad, la responsabilidad y sobre todo la solidaridad.

\section{Materiales y Métodos}

Se realizó un estudio observacional, descriptivo y bibliométrico de corte transversal evaluándose todas las investigaciones presentadas desde el 1ro de enero de 2020 y el 30 de mayo de 2021. Se valoraron los aspectos éticos que deberían cumplir y plasmarse en una investigación científica. en los artículos sobre COVID-19 ética e investigación. Se seleccionaron aquellas revistas de alto impacto a nivel regional, nacional e internacional, de ciencias médicas $(n=15)$. Para la recolección de la información se accedió al portal electrónico de las revistas desde el sitio web de Infomed (http://www.sld.cu/red-de-portales). El día 30 de mayo de 2021 se descargó cada artículo en sus formatos (PDF/HTML/XML) e idiomas disponibles (español/inglés), teniendo en cuenta volumen, número y sección al que pertenecía, autores con sus
Periodo. Julio - Diciembre 2021

Vol. 5, Nro. 2, Publicado: 2021-12-31

afiliaciones institucionales, lugar de origen $y$ cantidad de referencias bibliográficas.

El índice de Price fue calculado como la proporción de referencias con antigüedad $\leq 5$ años, según la fecha en que se recibió el artículo ( $\Sigma \mathrm{R} 5)$ y el total de referencias utilizadas ( $\Sigma \mathrm{TR})$ en el mismo. Además, se realizó ese mismo día una búsqueda en Google Académico empleando el título y los autores para determinar las citas que poseía cada artículo. Se analizó la tasa de variación (Tv), definida como la variación (incremento o disminución) de la producción científica, con respecto al mes anterior o al primer mes de un periodo. Se calcula $\mathrm{Tv}=[(\mathrm{Np}-$ $\left.\mathrm{Np} 0) \mathrm{Np}^{*} 100\right]$, donde $\mathrm{Np}$ es el total de artículos publicados en el último mes del periodo de análisis, Np0 el número total de artículos publicados en el primer mes del periodo de análisis. Los datos fueron almacenados en una base de datos confeccionada al efecto.

\section{Resultados}

De 15 revistas consultadas, 9 presentaron al menos un artículo sobre la COVID-19 (60 \%), para un total de 80 artículos. Las revistas con mayor número de artículos fueron Scielo y Dialnet $(33,75 \%)$ y MediSur $(26,25 \%)$

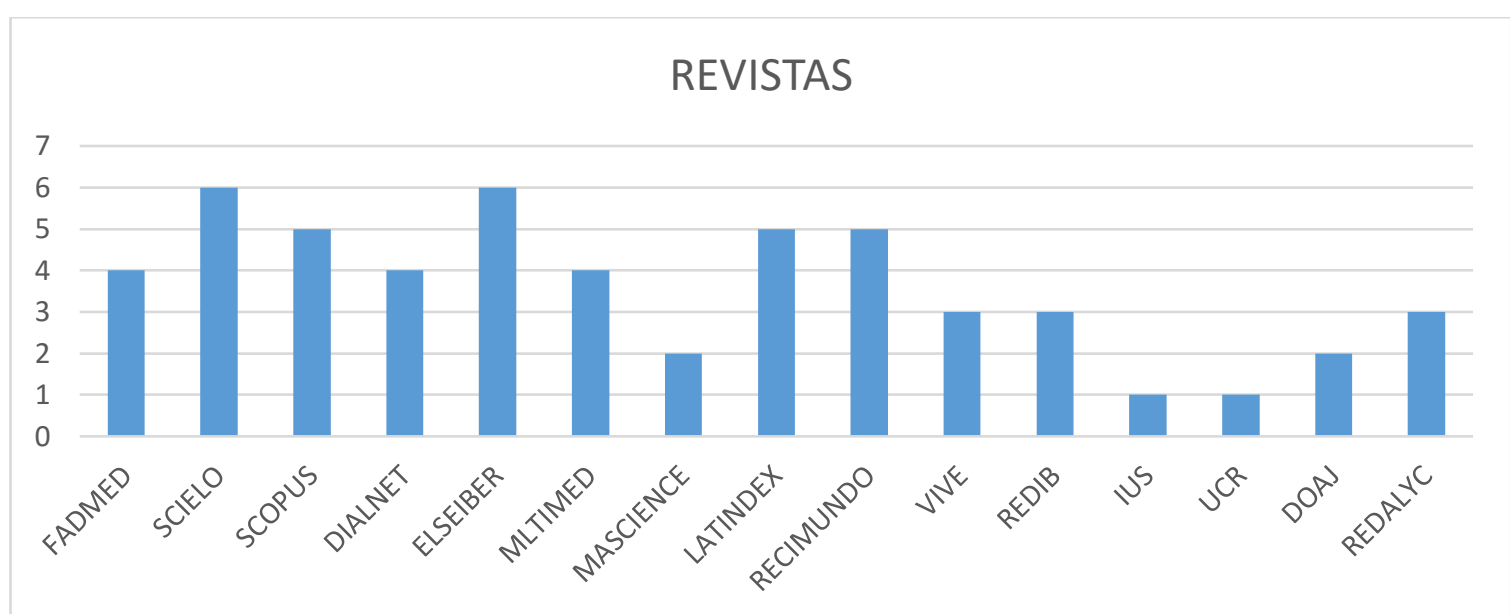


Fig. 1 Distribución de artículos sobre COVID-19 Ética e Investigación, publicados en las revistas de divulgación, enero-julio 2020.

Con respecto a los tipos de artículos, se encontró predominio de los artículos originales (40,25\%), seguidos de temas de actualidad $(28,57 \%)$ y artículos
Periodo. Julio - Diciembre 2021

Vol. 5, Nro. 2, Publicado: 2021-12-31 de revisión $(16,88 \%)$. El $(74,02) \%$ de los artículos fue publicado tanto en español como en idioma inglés, con predominio de temas de actualidad $(18,2$ \%) (Tabla 1). Los artículos en español e inglés correspondieron a Scopus y Scielo $(25,9 \%)$.

Tabla 1. Distribución de artículos según tipo e idioma

\begin{tabular}{|l|l|l|l|l|}
\hline \multirow{2}{*}{ Tipo de articulo } & Idioma & \multicolumn{2}{l|}{ Total } \\
\cline { 2 - 5 } & Español & Español e ingles & No. & $\%$ \\
\hline Articulo original & 27 & 4 & 31 & 40,25 \\
\hline Artículo de revisión & 8 & 5 & 13 & 16,88 \\
\hline Editorial & 3 & 2 & 5 & 6,49 \\
\hline Comunicación breve & 2 & - & 2 & 2,59 \\
\hline Temas de actualidad & 14 & 8 & 22 & 28,57 \\
\hline Articulo especial & 1 & - & 1 & 1,29 \\
\hline Manual & 2 & 1 & 3 & 3,89 \\
\hline Total & 57 & 20 & 77 & 100,0 \\
\hline
\end{tabular}

Tabla 2. El $100 \%$ de los artículos presentó autoría múltiple, dejando en evidencia la participación activa en de los investigadores. Se reportaron 99 autores, de ellos, el 85,85 \% de procedencia Internacional; y 14,15\% autorías nacionales (Tabla 2)

\begin{tabular}{|l|l|l|}
\hline Institución & No. Investigadores & $\%$ \\
\hline Centro Interdisciplinario de estudios en Bioética & 12 & 12,12 \\
\hline Comité de Ética de la Investigación con medicamentos de Galicia & 8 & 8.08 \\
\hline Clínica Estomatológica Docente & 4 & 4.04 \\
\hline Centro Universitario Newton Paiva & 16 & 16,16 \\
\hline Centro Universitario Curitiba & 4 & 4,04 \\
\hline Universidad de Ciencias Médicas Cienfuegos & 20 & 20,20 \\
\hline Universidad Autónoma del Estado de México, & 8 & 8,08 \\
\hline Universidad de Chile & 3 & 3,03 \\
\hline Universidad de Sao Paulo & 8 & 8,08 \\
\hline Universidad Central del Ecuador & 4 & 4,04 \\
\hline Universidad de Paivia & 4 & 4,04 \\
\hline
\end{tabular}


Periodo. Julio - Diciembre 2021

Vol. 5, Nro. 2, Publicado: 2021-12-31 Universidad de Federal de Minas Gerais, Belo Horizonte \begin{tabular}{|l|l}
\hline 8 & 8,08
\end{tabular}

Tabla 3.

\begin{tabular}{|c|c|c|c|c|c|}
\hline Revista & Vol. & Titulo & Autores & Sección & Idioma \\
\hline $\begin{array}{l}\text { Revista } \\
\text { enfermermería } \\
\text { Herediana }\end{array}$ & 2 & $\begin{array}{l}\text { Ética y Bioética del } \\
\text { nuevo siglo }\end{array}$ & $\begin{array}{l}\text { Guerrero Ramírez, Rosa, } \\
\text { Meneses La Riva María., } \\
\& \text { De La Cruz-Ruiz, } \\
\text { Marcy }\end{array}$ & $\begin{array}{l}\text { Articulo } \\
\text { original }\end{array}$ & Español \\
\hline $\begin{array}{l}\text { Revista } \\
\text { Colombiana de } \\
\text { Bioética }\end{array}$ & 1 & $\begin{array}{l}\text { Bioética } \\
\text { Humanización de los } \\
\text { Servicios de Salud }\end{array}$ & Andino Cancino & $\begin{array}{l}\text { Articulo } \\
\text { original }\end{array}$ & Español \\
\hline $\begin{array}{l}\text { Revista } \\
\text { Psicológica de } \\
\text { Chile }\end{array}$ & 23 & $\begin{array}{l}\text { Matrices Progresivas } \\
\text { de Raven: efecto Flynn } \\
\text { y actualización de } \\
\text { baremos. }\end{array}$ & $\begin{array}{l}\text { Rossi-Casé, Lilia; Neer, } \\
\text { Rosa; Lopetegui, Susana; } \\
\text { Doná, Stella; Biganzoli, } \\
\text { Bruno; Garzaniti, Ramiro }\end{array}$ & $\begin{array}{l}\text { Articulo } \\
\text { original }\end{array}$ & Español \\
\hline Revista Dialnet & 26 & $\begin{array}{l}\text { Humanización de la } \\
\text { asistencia médica en el } \\
\text { servicio de urgencias: } \\
\text { la importancia de los } \\
\text { valores en salud }\end{array}$ & Valentina Duarte & $\begin{array}{l}\text { Articulo } \\
\text { Original }\end{array}$ & Español \\
\hline Scielo & 10 & $\begin{array}{l}\text { La humanización de } \\
\text { (en) la Atención } \\
\text { Primaria. }\end{array}$ & $\begin{array}{l}\text { Rodrigo } \quad \text { Gutiérrez } \\
\text { Fernández }\end{array}$ & $\begin{array}{l}\text { Artículo } \\
\text { de } \\
\text { revisión }\end{array}$ & Español \\
\hline $\begin{array}{l}\text { Revista de la } \\
\text { Universidad } \\
\text { Industrial de } \\
\text { Santander. Salud }\end{array}$ & 47 & $\begin{array}{ll}\text { El cuidado de } \\
\text { enfermería a } \quad \text { los } \\
\text { Grupos Humanos }\end{array}$ & $\begin{array}{l}\text { María Isabel Lagoueyte } \\
\text { Gómez }\end{array}$ & $\begin{array}{l}\text { Artículo } \\
\text { de } \\
\text { revisión }\end{array}$ & Español \\
\hline Revista UNAL & 14 & $\begin{array}{l}\text { Motivos de uso de los } \\
\text { servicios de urgencias. }\end{array}$ & $\begin{array}{l}\text { Herney Alonso Rengifo } \\
\text { Reina, Patricia Rodríguez } \\
\text { Sánchez }\end{array}$ & $\begin{array}{l}\text { Artículo } \\
\text { de } \\
\text { revisión }\end{array}$ & Español \\
\hline Infomed & 19 & $\begin{array}{l}\text { Observancia de la ética } \\
\text { en investigaciones } \\
\text { presentadas en jornadas } \\
\text { científicas del hospital } \\
\text { clínico quirúrgico } \\
\text { Joaquín Albarrán }\end{array}$ & $\begin{array}{l}\text { Anabel Hernández Ruiz, } \\
\text { José Julián Castillo } \\
\text { Cuello, Marta Rodríguez } \\
\text { Acuña, Anabelis González } \\
\text { Hernández }\end{array}$ & $\begin{array}{l}\text { Articulo } \\
\text { origina; }\end{array}$ & Ingles \\
\hline
\end{tabular}




\section{Discusión}

La producción científica sobre ética e investigación es específica, variable e influenciada por diferentes fenómenos. El interés que la temática genera en el ecosistema científico, así como el nivel de especialización y experiencias alcanzadas en la temática, la inmediatez e impacto del tema hacen que los investigadores decidan investigar o no sobre la temática. En este caso, se puede destacar que la investigación sobre COVID-19 desde la parte ética, ha llamado la atención de investigadores de todos los campos de las ciencias de la salud.

La pandemia ha llevado la investigación y las publicaciones a un ritmo de avance sin precedentes. A junio de 2020 se habían publicado más de 17.700 artículos académicos sobre COVID-19 pero no todos abarcan el aspecto de tipo legal. Al ser esta una enfermedad polifacética y emergente, en la medida que se estudia, se descubren más afectaciones y surgen más preguntas que respuestas. Se necesita aún más tiempo e investigaciones para conocer todas las aristas clínicas y epidemiológicas de esta enfermedad, así como las repercusiones y secuelas a largo plazo. Por esto, se deben evitar las deducciones y conclusiones apresuradas. (5)

Al analizar la existencia de más 15 revistas encargadas de difundir los resultados investigativos de la ética en el contexto actual del Covid-19, se logra determinar de manera aleatoria la escasa divulgación de trabajos científicos. A pesar de la rigurosidad científica que exigen las investigaciones, particularmente en este campo de la salud, se ha desencadenado una frenética carrera por publicar resultados positivos sobre la COVID-19 en todo el mundo, muchos sin considerar la parte ética de sus investigaciones y publicaciones. Muchos de estos trabajos son informes preliminares que no han sido
Periodo. Julio - Diciembre 2021

Vol. 5, Nro. 2, Publicado: 2021-12-31

objeto de las profundas revisiones tradicionales, estudios inmaduros, sesgados o fraudulentos, lo que conlleva cuestionar la calidad científica de los trabajos y su credibilidad. (Hernandez Ruiz, Castillo Cuello, Rodriguez Acuna, \& Gonzalez Hernandez, 2020)

\section{Conclusiones}

La observancia de la ética en las investigaciones revisadas fue deficiente, por lo que es imprescindible realizar acciones de capacitación a investigadores, que intervienen directamente en la investigación con principios éticos. Futuros estudios deberían explorar los retos jurídicos y las herramientas, para continuar haciendo investigación. Sería importante hacer un seguimiento del desempeño y la producción científica para evaluar los verdaderos impactos que ha tenido esta crisis. Además. Para conocer el impacto de las políticas nacionales e internacionales impuestas como parte de la lucha contra el COVID19 es necesario ampliar la muestra a investigadores que realizan estudios de tipo experimental en todos los países.

La flexibilidad en cuanto a la ejecución de proyectos y la generación de expectativas más realistas en cuanto a los productos esperados podría ayudar a disminuir este sentimiento de incertidumbre y contribuir al avance de las investigaciones bajo las normativas de ética establecidas por la ONU. Varias acciones podrían influir positivamente en este sentido: los tiempos y rubros de los fondos asignados en los proyectos de investigación experimental ante el Covid-19 deberían ser extendidos; las expectativas de producción científica deberían ser consecuentes con las circunstancias, considerando las limitaciones y el tiempo. En cuanto a los estudiantes de tesis, se deberían implementar alternativas para que puedan tener opciones para culminar sus trabajos de 
Periodo. Julio - Diciembre 2021

titulación. Se deberían incluso establecer protocolos cuando este tipo de eventualidades sucedan.

A pesar de la amplitud del consenso alcanzado, este estudio deja a la vista muchos problemas éticos marginados de los artículos publicados. Así pues, el primer principio ético relativo al «respeto a la vida» no pudo ser integrado en los artículos dedicado a la dignidad humana y derechos humanos. Tampoco se evidencio los «derechos a un buen trato» en el

\section{Bibliografía}

De Arco-Canoles, O. D., \& Suarez-Calle, Z. K. (2018). Rol de los profesionales de enfermería en el sistema de salud colombiano. Universidad y Salud. Recuperado el 15 de 11 de 2020, de http://www.scielo.org.co/pdf/reus/v20n2/0124-7107-reus-20-02-00171.pdf

Guerrero Ramírez, R., Meneses La Riva, M. E., \& De La Cruz-Ruiz, M. (2015). Cuidado humanizado de enfermería según la teoría de Jean Watson,servicio de medicina del Hospital Daniel Alcides Carrión. Lima. Rev enferm Herediana, 10. Recuperado el 30 de 03 de 2021, de https://faenf.cayetano.edu.pe/images/2017/revistavol9/9.pdf Malpico Tinoco, M. C. (2017). PERCEPCIÓN DEL CUIDADO HUMANIZADO DE ENFERMERÍA POR PARTE DEL PACIENTE ADULTO CON ENFERMEDAD ONCOLÓGICA HOSPITALIZADO EN UNAIPS DE IV NIVEL. Mexico. Recuperado el 11 de 09 de 2020, de https://core.ac.uk/download/pdf/323265125.pdf Rodríguez Villalta, N. P. (2020). La bioetica en el contexto de la pandemia po COVID-19. Colombia. Recuperado el $10 \quad 09$ de $\quad 09$ de $2020, \quad$ https://alerta.salud.gob.sv/wpcontent/uploads/2020/07/editorial_la_bioetica_en_el_contexto_de_la_pandemia_por_covid-19_31_julio_4243.pdf

Andino, C. (2016). Bioetica y Humanización de los Servicios de Salud. Revista Colombiana de Bioetica, 29. Recuperado el 11 de 06 de 2021, de https://www.redalyc.org/jatsRepo/1892/189242405005/html/index.html Casé, L. R., \& Susana Lopetegu, R. N. (2014). Matrices Progresivas de Raven: efecto Flynn y actualización de baremos. Revista Psicologica de Chile, 12. Recuperado el 12 de 02 de 2021, de https://www.redalyc.org/pdf/264/26435341002.pdf

Consejeria de Sanidad. (2016). Plan de Humanizacion de asistencia sanitaria. Madrid: Biblioteca Virtual. Recuperado el 11 de 06 de 2021, de https://www.madrid.org/bvirtual/BVCM017902.pdf

Consejo de Organizaciones Internacionales de las Ciencias Medicas. (2016). Pautas éticas internacionales para la investigacion relacionada con la salud con seres vivos. Organizacion Mundial de la Salud, Ginebra. Recuperado el 3 de 06 de 2021, de https://iris.paho.org/bitstream/handle/10665.2/34457/9789290360902spa.pdf?sequence $=5 \&$ isAllowed $=\mathrm{y}$

Dadalto, L., Medeiros Royo, M., \& Silva Costa, B. (2020). Bioetica e integridad cientifica en la investigacion clinica sobre Covid-19. Scielo. Recuperado el 11 de 06 de 2021, de https://www.scielo.br/j/bioet/a/phw53Nvrf8bPqYhKqjX4Dvj/?lang=es 
Periodo. Julio - Diciembre 2021

Vol. 5, Nro. 2, Publicado: 2021-12-31

Duarte, V. (2014). Humanización de la asistencia médica en el servicio de urgencias: la importancia de los valores en salud. Dialnet, 16. Recuperado el 18 de 11 de 2020 , de https://dialnet.unirioja.es/servlet/articulo?codigo $=4911434$

Gutierrez Fernandez, R. (2017). La humanización de (en) la Atención Primaria. cielo, 11. Recuperado el 32 de 09 de 2020, de https://scielo.isciii.es/scielo.php?script=sci_arttext\&pid=S1699-695X2017000100005

Hernandez Ruiz, A., Castillo Cuello, J. J., Rodriguez Acuna, M., \& Gonzalez Hernandez, A. (2020). Observancia de la ética en investigaciones presentadas en jornadas científicas del hospital clínico quirúrgico Joaquín Albarrán. Infomed, 12. Recuperado el 06 de 14 de 2021, de http://www.revhabanera.sld.cu/index.php/rhab/article/view/3008 Lagoueyte Gómez, M. I. (2015). El cuidado de enfermería a los Grupos Humanos. Revista de la Universidad Industrial de Santander. Salud. Recuperado el 18 de 11 de 2020, de http://www.scielo.org.co/scielo.php?script=sci_arttext\&pid=S0121-08072015000200013

Maldonado Hurtado, S. M. (2018). Buenas practicas empresariales para la inclusion laboral de profesionales con discapacidad. Quito. Recuperado el $01 \quad$ de 02 de 2021, de http://repositorio.puce.edu.ec/bitstream/handle/22000/16072/DISERTACI\%C3\%93N_SHIRLEY\%20MICHELL E\%20MALDONADO.pdf?sequence=1\&isAllowed=y

Molano Gutiérrez, F. R., Escobar Grisales, C. M., García Vargas, M. A., Salazar Molina, P. A., Mejía Venegas, L. C., \& Jiménez Barbosa , W. G. (2016). Motivos de uso de los servicios de urgencias. ACTA. Odontologico $\begin{array}{llllllll}\text { Colombiano. } & \text { Recuperado } & \text { el } & 15 & \text { de } & 11 & \text { de } & 20202,\end{array}$ https://revistas.unal.edu.co/index.php/actaodontocol/article/view/58854

Organizacion Mundial de la Salud. (2020). COVID-19; Coronavirus; Ética en Investigación; Bioética. Organizacion Mundial de la Salud, Estados Unidos. Recuperado el 13 de 03 de 2021, de https://iris.paho.org/handle/10665.2/52142

Quintana Zavala , M. O. (2014). Calidad de vida en el tabajo, personal de enfermeria. Chile. Recuperado el 17 de 01

de 2021 ,

de

http://repositorio.udec.cl/bitstream/11594/1603/1/Tesis_Calidad_de_Vida_en_el_Trabajo.Image.Marked.pdf

Red de America Latinas Y el Caribe de Comites Nacionales de Bioetica. (2020). Ante las investigaciones biomédicas por la pandemia de enfermedad infecciosa por coronavirus Covid-19. Red de America Latinas Y el Caribe de Comites Nacionales de Bioetica, Estados Unidos. Recuperado el 23 de 03 de 2021, de file:///C:/Users/Usuario/Downloads/declaracion_red_alac_cnbs_investigaciones_covid_19.pdf

Rodriguez-Jimenez, ,. S., Cardenas-Jimenez, ,. M., Pacheco-Arce, A. L., \& Ramirez-Perez, ,. M. (2014). Una mirada fenomenológica del cuidado de enfermería. Scielo. Obtenido de http://www.scielo.org.mx/scielo.php?pid=S1665$70632014000400005 \&$ script=sci_abstract\&tlng=es

Santacruz-Bravo, J. D. (2015). Humanización de la calidad en la atención clínica en salud desde la perspectiva centrada en el paciente a partir de la Resolución. Colombia. Recuperado el 15 de 11 de 2020 , de http://www.scielo.org.co/pdf/reus/v18n2/v18n2a17.pdf 
Periodo. Julio - Diciembre 2021

Vol. 5, Nro. 2, Publicado: 2021-12-31

Savinovich Morán, C. (2014). Humanización en la atención de enfermería a los usuarios de la Sala de Emergencia del Hospital "Dr. Teodoro Maldonado Carbo", de diciembre 2013 a mayo 2014. Guayaquil. Recuperado el 01 de 02 de 2021, de http://repositorio.ucsg.edu.ec/handle/3317/3089

Valenzuela Anguita, M. (2015). Es posible humanizar los cuidados de enfermeria en los servicios de emergencias.

$\begin{array}{llllllll}\text { Madrid. } & \text { Recuperado } & \text { el } & 18 & \text { de } & 11 & \text { de } & 2020,\end{array}$ https://rua.ua.es/dspace/bitstream/10045/50212/1/tesis_martina_valenzuela_anguita.pdf 